\title{
RW Doradus: A solar-type shallow contact binary with a new orbital period investigation
}

\author{
Thawicharat SAROTSAKULCHAI (D), 1,2,3,* Sheng-Bang QIAN, ${ }^{1,2,4,5}$ \\ Boonrucksar SOONTHORNTHUM, ${ }^{3}$ Eduardo FeRnándeZ LAJÚs, ${ }^{6,7}$ \\ Nian-Ping Liu, ${ }^{1,4,5}$ Xiao Zhou, ${ }^{1,4,5}$ Jia ZhaNG, ${ }^{1,4,5}$ Wen-Ping LiaO, ${ }^{1,4,5}$ \\ Daniel E. ReICHART, ${ }^{8}$ Joshua B. HAISLIP, ${ }^{8}$ Vladimir V. KouPRIANOV, ${ }^{8}$ \\ and Saran POSHYACHINDA ${ }^{3}$
}

\author{
'Yunnan Observatories, Chinese Academy of Sciences, 650216 Kunming, China \\ ${ }^{2}$ University of Chinese Academy of Sciences, 19A Yuquan Road, Shijingshan, 100049 Beijing, China \\ ${ }^{3}$ National Astronomical Research Institute of Thailand, Ministry of Science and Technology, Bangkok, \\ Thailand \\ ${ }^{4}$ Key Laboratory of the Structure and Evolution of Celestial Objects, Chinese Academy of Sciences, 650216 \\ Kunming, China \\ ${ }^{5}$ Center for Astronomical Mega-Science, Chinese Academy of Sciences, 20A Datun Road, Chaoyang \\ District, Beijing, 100012, China \\ ${ }^{6}$ Facultad de Ciencias Astronómicas y Geofísicas - UNLP, 1900 La Plata, Buenos Aires, Argentina \\ ${ }^{7}$ Instituto de Astrofísica de La Plata - CONICET/UNLP, 1900 La Plata, Buenos Aires, Argentina \\ ${ }^{8}$ Department of Physics and Astronomy, University of North Carolina, CB \#3255, Chapel Hill, NC 27599, \\ USA \\ *E-mail: tavijarus@narit.or.th \\ Received 2018 November 1; Accepted 2018 December 12
}

\begin{abstract}
New CCD photometric light curves of the short-period ( $P=0.285 \mathrm{~d})$ eclipsing binary RW Dor are presented. The observations were performed with the PROMPT-8 robotic telescope at CTIO in Chile between 2015 March and 2017 March. Other eclipse timings were obtained from the $2.15 \mathrm{~m}$ JS telescope at CASLEO, San Juan, Argentina in 2011 December. Based on a light curve analysis, it is found that RW Dor is a W-type shallow contact binary with a fill-out factor $f \sim 11 \%$ and a high mass ratio $q \sim 1.587(1 / q=0.63)$, where the hotter component is the less massive one $\left(M_{1} \sim 0.52 M_{\odot}\right.$ and $\left.M_{2} \sim 0.82 M_{\odot}\right)$. For orbital-period investigation, 15 new eclipse times and those previously published were compiled. $O-C$ analysis with very weak evidence suggests that a long-term decrease in period with a rate of $d P / d t=-9.61 \times 10^{-9} \mathrm{~d} \mathrm{yr}^{-1}$ is superimposed on a cyclic variation ( $A_{3}=0.0054 \mathrm{~d}$ and $P_{3}=49.9 \mathrm{yr}$ ). The long-term decrease can be interpreted as mass transfer from the more massive component to the less massive one, or combined with angular momentum loss via magnetic braking. In addition, the marginal contact phase, high mass ratio $(1 / q>0.4)$, and long-term decrease in period all suggest that RW Dor is a newly formed contact binary via Case A mass transfer, and it will evolve into a deeper normal contact binary. If the cyclic change is correct, the light travel-time effect via the presence of a cool third body will be a more plausible explanation for this.
\end{abstract}


Key words: binaries: close — binaries: eclipsing — stars: evolution — stars: individual (RW Doradus)

\section{Introduction}

RW Dor (HD 269320, HIP 24763) is an important binary for studying the formation and evolution of a newly formed contact binary after the common convective envelope has been formed and in the transition either from detached or semi-detached into the contact phase, because there are few contact binaries that have been found to be newly formed or at the beginning of the contact phase that will evolve into normal contact binary stars when their mass ratio becomes higher, e.g., RV Psc (He \& Qian 2009) and V524 Mon (He et al. 2012). RW Dor is a short-period W UMa-type binary with an orbital period of $0.285 \mathrm{~d}$ that is very close to a new period distribution of EW-type contact binaries with a peak of $0.29 \mathrm{~d}$ published by Qian et al. (2017b), indicating that RW Dor is a typical W UMa-type contact binary that is in agreement with the report (Qian et al. 2017a). The system is near the Large Magellanic Cloud (LMC), but is not a member of the LMC as pointed out by Russo, Vittone, and Milano (1984) and Marino et al. (2007). RW Dor was discovered as a variable star by Leavitt (1908), and later classified as a W UMa-type eclipsing binary by Hertzsprung (1925). The first spectral classification of the variable was made by Cannon (1921), who gave a spectral type of K5. This was subsequently confirmed by McLaughlin (1927), who also classified the system as a late-type eclipsing binary with the same spectral type.

The first photographic times of light minima were reported by Hertzsprung (1928), who gave an orbital period of $0.143 \mathrm{~d}$. Later, eclipse times were obtained by many authors (e.g., Russo et al. 1984; Marton et al. 1989; Kaluzny \& Caillault 1989; Ogloza \& Zakrzewski 2004; Marino et al. 2007) and the linear ephemeris of the binary was also corrected. The complete light curves were analyzed with the Wilson-Devinney method (Wilson \& Devinney 1971) by Marton, Grieco, and Sistero (1989) and Kaluzny and Caillault (1989), who determined the photometric elements independently. Those solutions indicated that RW Dor belongs to a W-subtype contact binary with components not in poor thermal contact, as predicted by the thermal relaxation oscillation theory (Lucy \& Wilson 1979). Additionally, it was found that the light curves of RW Dor exhibited a significant difference in the depths of the minima and showed variation. Marton, Grieco, and Sistero (1989) explained the asymmetry in the light curves as a hot spot on the cooler and more massive component located near the neck connecting the stars, while Kaluzny and Caillault (1989) reported that their light curves were only marginally asymmetric and did not show any scatter beyond the observational errors.

The first radial velocity measurements of RW Dor were published by Hilditch, Hill, and Bell (1992) using the $3.9 \mathrm{~m}$ telescope of the Anglo-Australian Observatory. They found that RW Dor was composed of two K1type stars and determined a spectroscopic mass ratio of $q_{\mathrm{sp}}=0.68$. By combining the photometric solutions given by Kaluzny and Caillault (1989), Hilditch, Hill, and Bell (1992) derived the absolute parameters of the binary system. They confirmed that RW Dor is a W-subtype contact binary. Other radial velocity $V_{0}$ curves were obtained by Duerbeck and Rucinski (2007), who determined a spectroscopic mass ratio of $q_{\mathrm{sp}}=0.63$, which is close to that derived by Hilditch, Hill, and Bell (1992). However, they found $V_{0}=25 \mathrm{~km} \mathrm{~s}^{-1}$, which is somewhat smaller than the $V_{0}=66.5 \mathrm{~km} \mathrm{~s}^{-1}$ given by Hilditch, Hill, and Bell (1992). Marino et al. (2007) reported that some light curves of RW Dor exhibited asymmetry, being similar to the results published by Marton, Grieco, and Sistero (1989). They also recomputed the spectroscopic mass ratio by using the radial velocities given by Hilditch, Hill, and Bell (1992) and Duerbeck and Rucinski (2007). All the eclipse times were also reanalyzed, and they found a secular decrease in orbital period with a rate of $\Delta P / P \sim-6.3 \times 10^{-11}$. However, neither light curve variation nor the third light in the system have been found in recent publications (e.g., Deb \& Singh 2011).

In this paper we examine the variations of the light curve and determine new photometric solutions based on our CCD observations. Then, we compare our results with those from the other investigators. The orbital period changes are reinvestigated with new eclipse times together with the others collected from the literature. Finally, the formation and the evolutionary state of the system, as well as the probability of the additional companion orbiting around the contact binary, are all discussed.

\section{CCD photometric observations}

The first observations in the $V$-band with new times of two light minima were obtained with the $2.15 \mathrm{~m}$ "Jorge Sahade" (JS) telescope at Complejo Astronomico El Leoncito (CASLEO), San Juan, Argentina during 2011 December 9, 11, and 13. During the observations, a VersArray $1300 \mathrm{~B}$ camera was attached to the $2.15 \mathrm{~m}$ telescope, producing 1095, 1300, and 1000 images on 2011 December 9, 11, and 13, respectively. The second 
Table 1. Coordinates of RW Dor and the comparison and check stars.

\begin{tabular}{|c|c|c|c|c|c|c|c|c|}
\hline Target & Names & $\alpha_{\mathrm{J} 2000.0}$ & $\delta \mathrm{J} 2000.0$ & V & $R$ & $J$ & $H$ & K \\
\hline Variable star & RW Dor & $05^{\mathrm{h}} 18^{\mathrm{m}} 32^{\mathrm{s}} .5$ & $-68^{\circ} 13^{\prime} 32^{\prime \prime} .7$ & 11.16 & 8.66 & 9.282 & 8.781 & 8.709 \\
\hline Comparison star & GSC 09162-00441 & $05^{\mathrm{h}} 18^{\mathrm{m}} 43^{\mathrm{s}} .6$ & $-68^{\circ} 07^{\prime} 33^{\prime \prime} .7$ & 12.25 & 11.89 & 11.916 & 11.859 & 11.863 \\
\hline Check star & J05175264-6813241 & $05^{\mathrm{h}} 17^{\mathrm{m}} 52^{\mathrm{s}} .6$ & $-68^{\circ} 13^{\prime} 24^{\prime \prime} 1$ & 12.33 & 11.27 & 10.782 & 10.285 & 10.169 \\
\hline
\end{tabular}

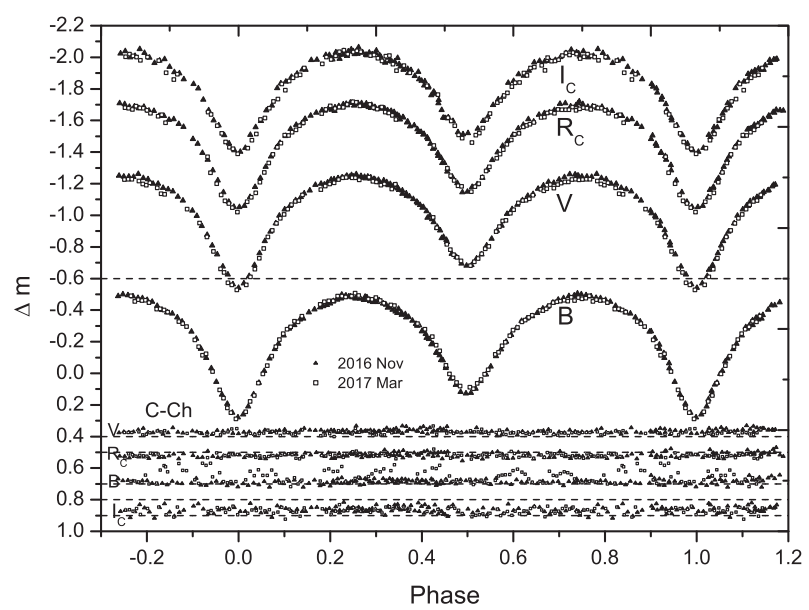

Fig. 1. Two sets for the second observation with complete light curves in the $B, V, R_{\mathrm{C}}$, and $I_{\mathrm{C}}$ bands were obtained with the $0.6 \mathrm{~m}$ telescope at CTIO in 2016 November (black triangles) and 2017 March (open squares). The differential magnitude between the comparison and check stars in the $B V R_{\mathrm{C}} I_{\mathrm{C}}$ bands $(\mathrm{C}-\mathrm{Ch})$ are plotted at the bottom of the figure and were used to calibrate all the data sets in each band.

observations in the $B, V, R_{\mathrm{C}} I_{\mathrm{C}}$ bands were obtained between 2015 March and 2017 March by using the back-illuminated Apogee F42 $2048 \times 2048$ CCD photometric system attached to the $0.60 \mathrm{~m}$ Cassegrain reflecting telescope of the PROMPT- $8^{1}$ robotic telescope, which is located at the Cerro Tololo Inter-American Observatory (CTIO) in Chile. It also provided nightly calibration images, including bias, dark, and flat-field images (Layden \& Broderick 2010). The CCD reduction and aperture photometry were done with standard procedure packages of IRAF. ${ }^{2}$ The comparison star (C) was GSC 09162-00441 [V $=12.25$, $J-H=0.057$ (SIMBAD)] and the check star (Ch) was 2MASS J05175264-6813241 $[\mathrm{V}=12.33, J-H=0.497$ (SIMBAD)]. The coordinates of those targets are listed in table 1 , and the complete multi-color light curves for the second observation in 2016 November and 2017 March are displayed by black triangles and open squares, respectively, in figure 1 . The data from figure 1 are listed in table 2 for 2016 and table 3 for 2017. A comparison of the two sets of light curves are also plotted together in the figure;

\footnotetext{
${ }^{1}$ PROMPT-8 is the Thai Southern Hemisphere Telescope (TST), operated in collaboration between the National Astronomical Research Institute of Thailand (NARIT) and the University of North Carolina (UNC) at Chapel Hill as part of the UNC-led PROMPT project 〈http://skynet.unc.edu〉.

2 The Image Reduction and Analysis Facility (IRAF): 〈http://iraf.noao.edu〉.
}

they nearly overlap, indicating that the light curves have no changes and are quite symmetric. This means that the light curves for our observations at this time are stable, which is the opposite of some results from previous investigators who found that the light curves showed asymmetry and varied with time. The depths of the primary and secondary minima of the two sets of light curves from figure 1 are given in table 4 , and no significant changes were found there. This helps to confirm that the light curves have no variations or asymmetry from the O'Connell effect (O'Connell 1951) caused by spot or magnetic activities which normally occur, being similar to the Sun or late-type stars, e.g., spectral type F, G, and K.

\section{Orbital period investigations}

The orbital period changes were analyzed using the $\mathrm{O}-\mathrm{C}$ value. All the available eclipse times from the literature were collected and investigated together with new CCD photometric times from the present observations as listed in table 5. The $\mathrm{O}-\mathrm{C}$ values of those eclipse times were computed using the linear ephemeris given by Kreiner (2004):

$\operatorname{Min} . \mathrm{I}(\mathrm{HJD})=2451869.076+0.2854633 E$,

where Min.I denotes the primary light minimum at phase $=$ 0.0 and $\mathrm{E}$ the cycle count after an estimated epoch. To analyze the $\mathrm{O}-\mathrm{C}$ changes, we first used a quadratic ephemeris to fit the $\mathrm{O}-\mathrm{C}$ curve with weight 1 for photographic data (pg) and weight 10 for photoelectric (pe) or CCD data. The result is plotted as the solid line in the upper panel of figure 2. A least-squares solution leads to the following quadratic ephemeris:

$$
\begin{aligned}
\operatorname{Min} . \mathrm{I}(\mathrm{HJD})= & 2451869.07751( \pm 0.00004) \\
& +0.285462985( \pm 0.000000002) E \\
& -\left[55.5( \pm 0.2) \times 10^{-13}\right] E^{2} .
\end{aligned}
$$

From the quadratic term in equation (2), the orbital period is decreasing and the change rate can be determined as $d P / d t=-14.19( \pm 0.05) \times 10^{-9} \mathrm{~d} \mathrm{yr}^{-1}$. The residuals from equation (2) are plotted in the lower panel of figure 2. As shown in figure 2, we found that a parabolic curve may not fit well; a cyclic variation seems to exist which can be seen clearly from the residuals in the lower panel that show a 
Table 2. CCD observations of RW Dor in 2016 November.

\begin{tabular}{|c|c|c|c|c|c|c|c|c|c|c|c|}
\hline $\begin{array}{c}\text { HJD } \\
(2457700+)\end{array}$ & $\Delta V$ & HJD & $\Delta R$ & HJD & $\Delta I$ & HJD & $\Delta V$ & HJD & $\Delta R$ & HJD & $\Delta I$ \\
\hline 20.5558 & -1.228 & 20.5562 & -1.641 & 20.5566 & -2.017 & 20.8129 & -1.061 & 20.8134 & -1.522 & 20.8138 & -1.894 \\
\hline 20.5585 & -1.233 & 20.5589 & -1.664 & 20.5593 & -1.991 & 20.8151 & -1.090 & 20.8155 & -1.531 & 20.8159 & -1.906 \\
\hline 20.5612 & -1.238 & 20.5616 & -1.681 & 20.5619 & -2.039 & 20.8172 & -1.099 & 20.8177 & -1.539 & 20.8180 & -1.918 \\
\hline 20.5637 & -1.250 & 20.5642 & -1.676 & 20.5646 & -2.037 & 20.8193 & -1.116 & 20.8198 & -1.557 & 20.8202 & -1.928 \\
\hline 20.5667 & -1.255 & 20.5672 & -1.692 & 20.5675 & -2.039 & 20.8213 & -1.117 & 20.8218 & -1.567 & 20.8223 & -1.921 \\
\hline 20.5696 & -1.260 & 20.5701 & -1.686 & 20.5706 & -2.023 & 20.8235 & -1.147 & 20.8240 & -1.580 & 20.8244 & -1.949 \\
\hline 20.5727 & -1.238 & 20.5731 & -1.675 & 20.5735 & -2.029 & 20.8267 & -1.158 & 20.8272 & -1.604 & 20.8276 & -1.965 \\
\hline 20.5756 & -1.234 & 20.5760 & -1.688 & 20.5764 & -2.012 & 20.8289 & -1.183 & 20.8294 & -1.633 & 20.8298 & -1.987 \\
\hline 20.5784 & -1.232 & 20.5789 & -1.659 & 20.5793 & -2.015 & 20.8307 & -1.194 & 20.8312 & -1.648 & 20.8316 & -1.990 \\
\hline 20.5814 & -1.225 & 20.5818 & -1.656 & 20.5822 & -2.042 & 21.5639 & -1.271 & 21.5643 & -1.709 & 21.5648 & -2.039 \\
\hline 20.5842 & -1.210 & 20.5845 & -1.656 & 20.5849 & -2.003 & 21.5668 & -1.265 & 21.5673 & -1.704 & 21.5677 & -2.054 \\
\hline 20.5868 & -1.220 & 20.5873 & -1.656 & 20.5877 & -1.966 & 21.5700 & -1.265 & 21.5704 & -1.687 & 21.5709 & -2.015 \\
\hline 20.5897 & -1.190 & 20.5902 & -1.630 & 20.5907 & -1.978 & 21.5729 & -1.264 & 21.5735 & -1.688 & 21.5739 & -2.024 \\
\hline 20.5926 & -1.175 & 20.5931 & -1.613 & 20.5936 & -1.973 & 21.5760 & -1.282 & 21.5763 & -1.677 & 21.5767 & -2.067 \\
\hline 20.5957 & -1.170 & 20.5961 & -1.608 & 20.5964 & -1.957 & 21.5789 & -1.256 & 21.5793 & -1.687 & 21.5798 & -2.042 \\
\hline 20.5984 & -1.137 & 20.5988 & -1.601 & 20.5991 & -1.921 & 21.5820 & -1.249 & 21.5824 & -1.663 & 21.5828 & -2.012 \\
\hline 20.6012 & -1.123 & 20.6017 & -1.573 & 20.6021 & -1.901 & 21.5851 & -1.220 & 21.5856 & -1.661 & 21.5860 & -1.979 \\
\hline 20.6080 & -1.077 & 20.6085 & -1.507 & 20.6089 & -1.861 & 21.5882 & -1.220 & 21.5887 & -1.629 & 21.5892 & -1.994 \\
\hline 20.6110 & -1.049 & 20.6115 & -1.491 & 20.6119 & -1.821 & 21.5914 & -1.194 & 21.5919 & -1.628 & 21.5923 & -2.003 \\
\hline 20.6140 & -0.984 & 20.6144 & -1.463 & 20.6148 & -1.815 & 21.5945 & -1.184 & 21.5950 & -1.605 & 21.5954 & -1.954 \\
\hline 20.6170 & -0.975 & 20.6175 & -1.401 & 20.6179 & -1.783 & 21.5975 & -1.172 & 21.5980 & -1.609 & 21.5984 & -1.921 \\
\hline 20.6201 & -0.927 & 20.6205 & -1.373 & 20.6208 & -1.709 & 21.6006 & -1.138 & 21.6011 & -1.579 & 21.6015 & -1.913 \\
\hline 20.6229 & -0.855 & 20.6233 & -1.311 & 20.6237 & -1.618 & 21.6036 & -1.114 & 21.6040 & -1.560 & 21.6043 & -1.876 \\
\hline 20.6259 & -0.845 & 20.6264 & -1.256 & 20.6267 & -1.613 & 21.6095 & -1.050 & 21.6100 & -1.483 & 21.6103 & -1.846 \\
\hline 20.6291 & -0.757 & 20.6295 & -1.223 & 20.6298 & -1.597 & & -1.016 & & -1.453 & 21.6133 & -1.818 \\
\hline 20.6321 & -0.735 & 20.6326 & -1.184 & 20.6329 & -1.526 & 21.6156 & -0.952 & 21.6159 & -1.394 & 21.6164 & -1.746 \\
\hline 20.6352 & -0.694 & 20.6357 & -1.126 & 20.6361 & -1.512 & 21.6188 & -0.897 & 21.6192 & -1.351 & 21.6197 & -1.680 \\
\hline 20.6384 & -0.682 & 20.6388 & -1.130 & 20.6393 & -1.520 & 21.6219 & -0.828 & 21.6224 & -1.285 & 21.6228 & -1.615 \\
\hline 20.6414 & -0.686 & 20.6419 & -1.160 & 20.6423 & -1.480 & 21.6250 & -0.772 & 21.6255 & -1.220 & 21.6259 & -1.584 \\
\hline 20.6446 & -0.727 & 20.6451 & -1.177 & 20.6454 & -1.548 & & -0.696 & & -1.145 & 21.6295 & -1.522 \\
\hline 20.6477 & -0.752 & 20.6483 & -1.217 & 20.6486 & -1.597 & 21.6317 & -0.645 & 21.6322 & -1.085 & 21.6325 & -1.470 \\
\hline 20.6509 & -0.809 & 20.6513 & -1.268 & 20.6517 & -1.624 & 21.6349 & -0.583 & 21.6354 & -1.055 & 21.6358 & -1.427 \\
\hline 20.6540 & -0.852 & 20.6545 & -1.330 & 20.6549 & -1.698 & 21.6384 & -0.561 & 21.6389 & -1.052 & 21.6394 & -1.417 \\
\hline 20.6573 & -0.902 & 20.6576 & -1.379 & 20.6581 & -1.733 & 21.6417 & -0.590 & 21.6421 & -1.059 & 21.6425 & -1.441 \\
\hline 20.6603 & -0.935 & 20.6608 & -1.419 & 20.6612 & -1.751 & 21.6450 & -0.636 & 21.6455 & -1.116 & 21.6458 & -1.524 \\
\hline 20.6636 & -1.010 & 20.6639 & -1.450 & 20.6644 & -1.838 & 21.6482 & -0.706 & 21.6487 & -1.179 & 21.6491 & -1.573 \\
\hline 20.6668 & -1.040 & 20.6673 & -1.489 & 20.6677 & -1.850 & 21.6515 & -0.780 & 21.6519 & -1.235 & 21.6524 & -1.613 \\
\hline 20.6701 & -1.068 & 20.6706 & -1.530 & 20.6709 & -1.892 & 21.6548 & -0.857 & 21.6553 & -1.306 & 21.6557 & -1.680 \\
\hline 20.6732 & -1.096 & 20.6737 & -1.552 & 20.6741 & -1.883 & 21.6582 & -0.917 & 21.6586 & -1.373 & 21.6591 & -1.710 \\
\hline 20.6765 & -1.123 & 20.6769 & -1.589 & 20.6773 & -1.915 & 21.6616 & -0.970 & 21.6621 & -1.430 & 21.6625 & -1.819 \\
\hline 20.6797 & -1.134 & 20.6802 & -1.574 & 20.6806 & -1.968 & 21.6637 & -1.017 & 21.6642 & -1.459 & 21.6646 & -1.849 \\
\hline 20.6830 & -1.168 & 20.6835 & -1.616 & 20.6839 & -1.978 & 21.6670 & -1.058 & 21.6675 & -1.501 & 21.6678 & -1.862 \\
\hline 20.6863 & -1.188 & 20.6868 & -1.625 & 20.6870 & -1.973 & 21.6703 & -1.080 & 21.6708 & -1.547 & 21.6713 & -1.892 \\
\hline 20.6894 & -1.212 & 20.6899 & -1.632 & 20.6904 & -1.978 & 21.6738 & -1.116 & 21.6742 & -1.572 & 21.6747 & -1.911 \\
\hline 20.6928 & -1.205 & 20.6932 & -1.646 & 20.6936 & -1.982 & 21.6773 & -1.135 & 21.6778 & -1.583 & 21.6782 & -1.938 \\
\hline 20.6961 & -1.227 & 20.6966 & -1.685 & 20.6970 & -2.002 & 21.6793 & -1.159 & 21.6797 & -1.610 & 21.6802 & -1.936 \\
\hline 20.6994 & -1.242 & 20.6998 & -1.690 & 20.7002 & -2.026 & 21.6827 & -1.177 & 21.6832 & -1.631 & 21.6837 & -1.998 \\
\hline 20.7026 & -1.260 & 20.7031 & -1.669 & 20.7036 & -2.048 & 21.6863 & -1.213 & 21.6868 & -1.649 & 21.6872 & -2.000 \\
\hline 20.7060 & -1.259 & 20.7065 & -1.676 & 20.7069 & -2.031 & 21.6898 & -1.221 & 21.6903 & -1.664 & 21.6906 & -1.957 \\
\hline 20.7080 & -1.251 & 20.7085 & -1.700 & 20.7089 & -2.006 & 21.6917 & -1.214 & 21.6922 & -1.663 & 21.6926 & -2.030 \\
\hline 20.7538 & -1.013 & 20.7542 & -1.485 & 20.7546 & -1.814 & 21.6938 & -1.228 & 21.6944 & -1.675 & 21.6948 & -2.030 \\
\hline 20.7563 & -0.987 & 20.7568 & -1.419 & 20.7573 & -1.777 & 21.6959 & -1.247 & 21.6964 & -1.668 & 21.6969 & -2.002 \\
\hline 20.7591 & -0.960 & 20.7596 & -1.378 & 20.7601 & -1.749 & 21.6979 & -1.242 & 21.6985 & -1.683 & 21.6989 & -2.047 \\
\hline 20.7617 & -0.906 & 20.7621 & -1.355 & 20.7625 & -1.703 & 21.7003 & -1.254 & 21.7008 & -1.691 & 21.7013 & -2.032 \\
\hline
\end{tabular}


Table 2. (Continued)

\begin{tabular}{ccccccccccccccc}
\hline $\begin{array}{c}\mathrm{HJD} \\
(2457700+)\end{array}$ & $\Delta V$ & $\mathrm{HJD}$ & $\Delta R$ & $\mathrm{HJD}$ & $\Delta I$ & $\mathrm{HJD}$ & $\Delta V$ & $\mathrm{HJD}$ & $\Delta R$ & $\mathrm{HJD}$ & $\Delta I$ & \\
\hline 20.7651 & -0.828 & 20.7656 & -1.299 & 20.7660 & -1.615 & 21.7028 & -1.260 & 21.7032 & -1.685 & 21.7037 & -2.031 \\
20.7895 & -0.656 & 20.7900 & -1.141 & 20.7904 & -1.495 & 21.7050 & -1.261 & 21.7055 & -1.705 & 21.7059 & -2.024 \\
20.7915 & -0.676 & 20.7920 & -1.153 & 20.7923 & -1.530 & 21.7075 & -1.263 & 21.7081 & -1.713 & 21.7084 & -2.065 \\
20.7937 & -0.724 & 20.7942 & -1.199 & 20.7946 & -1.558 & 21.7099 & -1.265 & 21.7104 & -1.694 & 21.7108 & -2.035 \\
20.7970 & -0.808 & 20.7975 & -1.254 & 20.7980 & -1.643 & 21.7122 & -1.265 & 21.7127 & -1.709 & 21.7131 & -2.078 \\
20.8002 & -0.869 & 20.8007 & -1.330 & 20.8010 & -1.710 & 21.7145 & -1.268 & 21.7150 & -1.709 & 21.7155 & -2.047 \\
\hline
\end{tabular}

large systematic scatter. Therefore, to get a better fit for the trend of $\mathrm{O}-\mathrm{C}$, a sinusoidal term is added to a quadratic ephemeris. By using a least-squares method, the ephemeris is redetermined as

$\operatorname{Min} . \mathrm{I}(\mathrm{HJD})=2451869.07786( \pm 0.00004)$

$$
\begin{aligned}
& +0.285463214( \pm 0.000000007) E \\
& -\left[37.6( \pm 0.5) \times 10^{-13}\right] E^{2} \\
& +0.0054( \pm 0.0001) \\
& \times \sin [0.00564 E+185.824( \pm 0.684)]
\end{aligned}
$$

As shown in figure 3, equation (3) can give a good description of the general trend of the $O-C$ curve. After the downward parabolic change is subtracted, the cyclic oscillation is displayed in the middle panel. From this ephemeris, the sinusoidal term suggests that the semiamplitude of cyclic variation is about $0.0054 \mathrm{~d}$, while it has a long period of $49.92 \mathrm{yr}$. The quadratic term also reveals a continuous decrease at a rate of $d P / d t=$ $-9.61( \pm 0.13) \times 10^{-9} \mathrm{dyr}^{-1}$ that is smaller than that derived from equation (2). The residuals from equation (3) are also plotted in the bottom panel of figure 3. Its scatters are smaller than those in figure 2, which can be justified by the sum of weighted squares deviation of 0.000851 for equation (2) without a cyclical term and 0.000462 for equation (3) with a cyclical term, respectively.

However, it cannot be concluded that the result from figure 3 is better and more reliable than figure 2 because of the small number of $\mathrm{O}-\mathrm{C}$ data that do not cover (the gap between 1943 and 1980 or about $36.64 \mathrm{yr}$ ) the whole cycles. Furthermore, the period of the third body from figure 3 is $49.92 \mathrm{yr}$, while the total time span of available eclipse times is only $127.48 \mathrm{yr}$. Besides, the first nine minima (pre-1944) are photographic (pg), listed in table 3 to only three decimal places, and thus they have ten times smaller weights when compared to the photoelectric (pe) and CCD data. Consequently, the effective time span of higher-quality minima (pe and CCD data) is only $37 \mathrm{yr}$, less than the third body orbit period. Moreover, the point density ( 9 minima over a time interval of $53.81 \mathrm{yr}$ ) for the first group is much lower than that of the second group (69 minima over a time interval of $37 \mathrm{yr}$ ). For these reasons, the evidence for a sinusoidal term in figure 3 is very weak. The result from equation (2) may be a possible explanation of the period change in the RW Dor system. However, to check the period changes proposed here, more eclipse times are still required in the future to confirm the period variations.

\section{Photometric solutions}

As shown in figure 1, the two sets of light curves obtained in 2016 November and 2017 March nearly overlap. This suggests that the light curves are stable within the error at that time interval, and no light curve variations from the O'Connell effect or spot activity cycles are found. However, the light curves of some close binaries are asymmetric, which could be explained by spot activity on one components or both (e.g., Qian et al. 2017a). For RW Dor, the light curves are quite symmetric indicating that they are very useful in determining the photometric solutions with high accuracy so that the more complicated solution of a spot model is not needed. We model the two sets of light curves separately: set- 1 refers to the one observed on 2016 November 28-29 and set- 2 refers to the other obtained on 2017 March 14-15. The two data sets in $B, V, R_{\mathrm{C}}$, and $I_{\mathrm{C}}$ bands were analyzed separately by using the Wilson and Devinney (W-D) code (Wilson \& Devinney 1971; Wilson 1990, 1994, 2012; van Hamme \& Wilson 2007).

Hilditch, Hill, and Bell (1992) classified the spectral type of the binary as $\mathrm{K} 1 \mathrm{~V}$ following Marton, Grieco, and Sistero (1989). The Tycho-2 mean color index $B-V=0.66$ (Høg et al. 2000) corresponds to a spectral type of G4/5 V, while the color index in the SIMBAD database reveals a $B-V=0.69$. For our solutions, the effective temperature of the primary star $\left(T_{1}\right)$ was assumed to be $5560 \mathrm{~K}$ according to its spectral type (Cox 2000). We assume that the convective envelopes are already developed. Thus, the bolometric albedos for stars 1 and 2 were taken as $A_{1}=$ $A_{2}=0.5$, and the values of the gravity-darkening coefficients $g_{1}=g_{2}=0.32$ were used. The monochromatic and bolometric limb-darkening coefficients were taken with a logarithmic law from van Hamme's table (van Hamme 
Table 3. CCD observations of RW Dor in 2017 March.

\begin{tabular}{|c|c|c|c|c|c|c|c|c|c|c|c|}
\hline $\begin{array}{c}\text { HJD } \\
+2457800\end{array}$ & $\Delta V$ & HJD & $\Delta R$ & HJD & $\Delta I$ & HJD & $\Delta V$ & HJD & $\Delta R$ & HJD & $\Delta I$ \\
\hline 26.5331 & -0.871 & 26.5335 & -1.338 & 26.5339 & -1.683 & 26.6569 & -1.095 & 26.6433 & -1.626 & 26.6699 & -1.719 \\
\hline 26.5353 & -0.837 & 26.5356 & -1.290 & 26.5359 & -1.663 & 26.6645 & -1.007 & 26.6695 & -1.410 & 26.6717 & -1.703 \\
\hline 26.5374 & -0.806 & 26.5378 & -1.261 & 26.5381 & -1.633 & 26.6691 & -0.919 & 26.6713 & -1.310 & 27.5323 & -1.642 \\
\hline 26.5396 & -0.789 & 26.5401 & -1.227 & 26.5404 & -1.575 & 26.6731 & -0.826 & 27.5319 & -1.251 & 27.5350 & -1.593 \\
\hline 26.5420 & -0.766 & 26.5424 & -1.223 & 26.5428 & -1.553 & 27.5342 & -0.725 & 27.5346 & -1.179 & 27.5373 & -1.540 \\
\hline 26.5479 & -0.737 & 26.5484 & -1.190 & 26.5487 & -1.525 & 27.5365 & -0.703 & 27.5370 & -1.142 & 27.5397 & -1.506 \\
\hline 26.5503 & -0.761 & 26.5507 & -1.221 & 26.5511 & -1.572 & 27.5388 & -0.638 & 27.5393 & -1.124 & 27.5420 & -1.497 \\
\hline 26.5526 & -0.781 & 26.5530 & -1.240 & 26.5534 & -1.586 & 27.5412 & -0.625 & 27.5416 & -1.098 & 27.5450 & -1.464 \\
\hline 26.5548 & -0.811 & 26.5552 & -1.282 & 26.5556 & -1.617 & 27.5443 & -0.593 & 27.5447 & -1.070 & 27.5511 & -1.499 \\
\hline 26.5571 & -0.843 & 26.5575 & -1.320 & 26.5579 & -1.671 & 27.5503 & -0.624 & 27.5507 & -1.136 & 27.5537 & -1.551 \\
\hline 26.5593 & -0.859 & 26.5596 & -1.347 & 26.5600 & -1.709 & 27.5529 & -0.675 & 27.5533 & -1.164 & 27.5564 & -1.589 \\
\hline 26.5639 & -0.951 & 26.5642 & -1.402 & 26.5646 & -1.778 & 27.5556 & -0.724 & 27.5561 & -1.192 & 27.5591 & -1.681 \\
\hline 26.5659 & -0.983 & 26.5662 & -1.428 & 26.5666 & -1.791 & 27.5583 & -0.794 & 27.5588 & -1.259 & 27.5619 & -1.691 \\
\hline 26.5680 & -1.020 & 26.5684 & -1.456 & 26.5688 & -1.823 & 27.5611 & -0.855 & 27.5615 & -1.322 & 27.5646 & -1.753 \\
\hline 26.5700 & -1.023 & 26.5705 & -1.490 & 26.5708 & -1.840 & 27.5637 & -0.929 & 27.5642 & -1.381 & 27.5672 & -1.795 \\
\hline 26.5728 & -1.068 & 26.5732 & -1.518 & 26.5736 & -1.879 & 27.5665 & -0.969 & 27.5668 & -1.436 & 27.5699 & -1.811 \\
\hline 26.5748 & -1.091 & 26.5753 & -1.553 & 26.5756 & -1.903 & 27.5691 & -1.014 & 27.5695 & -1.481 & 27.5722 & -1.907 \\
\hline 26.5776 & -1.134 & 26.5781 & -1.558 & 26.5784 & -1.906 & 27.5714 & -1.046 & 27.5719 & -1.507 & 27.5746 & -1.867 \\
\hline 26.5804 & -1.148 & 26.5808 & -1.600 & 26.5812 & -1.965 & 27.5738 & -1.071 & 27.5742 & -1.557 & 27.5769 & -1.924 \\
\hline 26.5824 & -1.150 & 26.5828 & -1.609 & 26.5831 & -1.950 & 27.5761 & -1.105 & 27.5765 & -1.556 & 27.5792 & -1.946 \\
\hline 26.5845 & -1.160 & 26.5849 & -1.617 & 26.5853 & -1.997 & 27.5784 & -1.132 & 27.5789 & -1.576 & 27.5815 & -1.961 \\
\hline 26.5868 & -1.182 & 26.5872 & -1.627 & 26.5876 & -2.002 & 27.5808 & -1.152 & 27.5811 & -1.591 & 27.5838 & -1.965 \\
\hline 26.5916 & -1.219 & 26.5919 & -1.659 & 26.5923 & -2.014 & 27.5830 & -1.159 & 27.5834 & -1.617 & 27.5863 & -2.007 \\
\hline 26.5935 & -1.211 & 26.5940 & -1.661 & 26.5942 & -1.983 & 27.5853 & -1.194 & 27.5858 & -1.636 & 27.5975 & -2.034 \\
\hline 26.5954 & -1.224 & 26.5958 & -1.687 & 26.5960 & -2.063 & 27.5967 & -1.228 & 27.5971 & -1.693 & 27.6002 & -2.076 \\
\hline 26.5976 & -1.250 & 26.5980 & -1.694 & 26.5984 & -2.041 & 27.5994 & -1.250 & 27.5998 & -1.720 & 27.6030 & -2.061 \\
\hline 26.5998 & -1.227 & 26.6002 & -1.686 & 26.6006 & -2.043 & 27.6021 & -1.264 & 27.6025 & -1.743 & 27.6058 & -2.084 \\
\hline 26.6022 & -1.250 & 26.6025 & -1.710 & 26.6028 & -2.049 & 27.6049 & -1.270 & 27.6054 & -1.725 & 27.6083 & -2.110 \\
\hline 26.6044 & -1.258 & 26.6049 & -1.708 & 26.6052 & -2.076 & 27.6077 & -1.292 & 27.6081 & -1.736 & 27.6111 & -2.120 \\
\hline 26.6067 & -1.266 & 26.6071 & -1.708 & 26.6074 & -2.093 & 27.6102 & -1.302 & 27.6107 & -1.733 & 27.6133 & -2.095 \\
\hline 26.6089 & -1.266 & 26.6092 & -1.709 & 26.6096 & -2.079 & 27.6125 & -1.304 & 27.6130 & -1.752 & 27.6169 & -2.108 \\
\hline 26.6110 & -1.282 & 26.6114 & -1.725 & 26.6117 & -2.094 & 27.6162 & -1.302 & 27.6165 & -1.768 & 27.6191 & -2.115 \\
\hline 26.6132 & -1.283 & 26.6137 & -1.705 & 26.6140 & -2.086 & 27.6183 & -1.302 & 27.6187 & -1.765 & 27.6214 & -2.110 \\
\hline 26.6155 & -1.279 & 26.6159 & -1.714 & 26.6162 & -2.057 & 27.6206 & -1.290 & 27.6211 & -1.742 & 27.6276 & -2.085 \\
\hline 26.6177 & -1.282 & 26.6182 & -1.721 & 26.6186 & -2.069 & 27.6268 & -1.307 & 27.6272 & -1.752 & 27.6299 & -2.090 \\
\hline 26.6201 & -1.284 & 26.6204 & -1.718 & 26.6208 & -2.085 & 27.6291 & -1.280 & 27.6295 & -1.731 & 27.6322 & -2.069 \\
\hline 26.6223 & -1.283 & 26.6228 & -1.711 & 26.6231 & -2.074 & 27.6314 & -1.277 & 27.6318 & -1.722 & 27.6349 & -2.052 \\
\hline 26.6268 & -1.272 & 26.6249 & -1.730 & 26.6253 & -2.066 & 27.6341 & -1.256 & 27.6346 & -1.735 & 27.6371 & -2.082 \\
\hline 26.6291 & -1.253 & 26.6273 & -1.719 & 26.6276 & -2.046 & 27.6363 & -1.264 & 27.6367 & -1.703 & 27.6393 & -2.085 \\
\hline 26.6313 & -1.246 & 26.6295 & -1.718 & 26.6321 & -2.066 & 27.6408 & -1.232 & 27.6389 & -1.698 & 27.6415 & -2.032 \\
\hline 26.6336 & -1.261 & 26.6317 & -1.704 & 26.6391 & -2.044 & 27.6430 & -1.261 & 27.6413 & -1.682 & 27.6438 & -1.990 \\
\hline 26.6382 & -1.229 & 26.6340 & -1.686 & 26.6437 & -1.967 & 27.6666 & -1.052 & 27.6435 & -1.656 & 27.6484 & -2.022 \\
\hline 26.6406 & -1.192 & 26.6387 & -1.667 & 26.6485 & -1.925 & 27.6689 & -0.987 & 27.6480 & -1.720 & 27.6521 & -1.977 \\
\hline 26.6428 & -1.188 & 26.6410 & -1.677 & 26.6507 & -1.957 & & & & & & \\
\hline
\end{tabular}

1993). All the fixed parameters are listed in table 4. The adjustable parameters are the inclination $(i)$, the mass ratio $(q)$, the temperature of star $2\left(T_{2}\right)$, the monochromatic luminosity of star $1\left(L_{1 B}, L_{1 V}, L_{1 R_{C}}\right.$, and $\left.L_{1 I_{\mathrm{C}}}\right)$, and the dimensionless potential of star $1\left(\Omega_{1}=\Omega_{2}\right)$ in mode 3 for contact configuration.
For a precise mass ratio determination, Hilditch, Hill, and Bell (1992) published results of radial-velocity measurements and gave a mass ratio of $q_{\mathrm{sp}}=0.68 \pm 0.03$. Later, Duerbeck and Rucinski (2007) obtained $q_{\mathrm{sp}}=0.63$ \pm 0.03 . However, we used a $q$-search method first with our photometric data to determine the photometric mass ratio 
Table 4. Depths of eclipse (differential magnitude) in each band.

\begin{tabular}{lcc}
\hline Filters & $2016 \mathrm{Nov}(\mathrm{pri}, \mathrm{sec})$ & $2017 \mathrm{Mar}$ (pri, sec) \\
\hline$\Delta B( \pm 0.004)$ & $0.280,0.127$ & $0.288,0.089$ \\
$\Delta V( \pm 0.002)$ & $-0.541,-0.682$ & $-0.536,-0.681$ \\
$\Delta R_{C}( \pm 0.002)$ & $-1.052,-1.158$ & $-1.033,-1.151$ \\
$\Delta I_{C}( \pm 0.003)$ & $-1.402,-1.491$ & $-1.389,-1.487$ \\
\hline
\end{tabular}

$\left(q_{\mathrm{ph}}\right)$ and then set the mass ratio as an adjustable parameter to get a better fit for the data in set- 1 and set- 2 . We obtained the initial mass ratio at $q=1.6(q>1.0$ for W-subtype) and then the differential correction was performed until the final solutions were derived at the lowest sum of the weighted square deviations $\Sigma[W(O-C)]^{2}$, hereafter $\Sigma$. The result is about $q$ (W-subtype $)=1.62 \pm 0.02$ or $q=0.615$ in general meaning-which is close to the spectroscopic mass ratio of 0.63 derived by Duerbeck and Rucinski (2007)—for both data sets when the more massive component of the W-subtype system is the cooler. However, it is obvious that both sets of light curves show partial eclipses $\left(i<85^{\circ}\right)$ so that their photometric mass ratios obtained by $q$-search may not be accurate, as discussed by Terrell and Wilson (2005). Therefore, we used the spectroscopic mass ratio of 0.63 or $q$ (W-subtype $)=1.587$ for our fixed mass ratio in the modeling process. The lightcurve modeling results with optimum parameters are listed in table 6. The corresponding theoretical light curves (solid lines) were compared with the observed light curves as shown in figure 4 for the data in set- 1 (left panel) and set- 2 (right panel). Additionally, figure 3 shows a cyclic variation that may be caused by the light travel-time effect via the presence of a third body. Therefore, we added the third light $\left(l_{3}\right)$ as an adjustable parameter in order to check the luminosity contribution of such a third companion, but the results show negative values for both data sets. This may suggest that if the third body really exists, it will be a very cool stellar companion. On the other hand, it may have no companion orbiting the eclipsing pair. The presence of the third body is discussed again in the next section. The geometrical structures of RW Dor based on the modeling are displayed in figure 5 .

\section{Discussions and conclusions}

The two sets of complete multi-color light curves in the $B V R_{C} I_{C}$ bands were obtained by using the PROMPT- 8 robotic telescope at CTIO in Chile between 2015 March and 2017 March. The other data in 2011 were obtained by using the $2.15 \mathrm{~m}$ telescope at CASLEO, San Juan, in Argentina. We compared our results with the light curves published by Deb and Singh (2011): no O'Connell effect or light curve variations were found. The photometric solutions indicate that RW Dor is a W-subtype, shallow contact binary with a degree of contact more than $10 \%$ and a high mass ratio $q$ (W-subtype) $\sim 1.587$ or $q=0.63$ in general meaning, which indicates that the hotter component is the less massive one. The absolute dimensions of RW Dor derived by using our photometric elements together with spectroscopic ones by Duerbeck and Rucinski (2007) are: $M_{1}=0.52 M_{\odot}, M_{2}=$ $0.82 \mathrm{M}_{\odot}, a=2.03 R_{\odot}, R_{1}=0.703 R_{\odot}, R_{2}=0.881 R_{\odot}$, $L_{1}=0.423 L_{\odot}$, and $L_{2}=0.534 L_{\odot}$. These parameters are close to those recently derived by Deb and Singh (2011).

The downward parabolic curve in the $\mathrm{O}-\mathrm{C}$ diagram shows an orbital period decrease at a rate of $d P / d t$ $=-14.19 \times 10^{-9} \mathrm{dyr}^{-1}$ without a sinusoidal term and $d P / d t=-9.61 \times 10^{-9} \mathrm{dyr}^{-1}$ with a cyclical term. The type of variations, i.e., a long-term decrease combined with a cyclic change, is commonly found in W UMa-type stars, for example V417 Aql (Qian 2003), V1139 Cas (Li et al. 2015b), MR Com (Qian et al. 2013b), BX Peg (Li et al. 2015a), V524 Mon (He et al. 2012), and V1073 Cyg (Tian et al. 2018). Some W-type contact binaries whose properties are similar to RW Dor are listed in table 8: most of them are shallow contact binaries with decreasing period. The long-term decrease in period can be explained either by mass transfer from the more massive component to the less massive one or by angular momentum loss (AML) via magnetic braking, or by a combination of both processes. If the long-term decrease in period is due to conservative mass transfer, the mass transfer rate can be determined with the following equation (Kwee 1958):

$$
\frac{\dot{P}}{P}=-3 \dot{M}_{2}\left(\frac{1}{M_{1}}-\frac{1}{M_{2}}\right)
$$

The mass transfer rate is $d M / d t=23.55 \times 10^{-9} M_{\odot} \mathrm{yr}^{-1}$ without a cyclical term and $d M / d t=15.95 \times 10^{-9} M_{\odot} \mathrm{yr}^{-1}$ with a cyclical term. The timescale of mass transfer can be computed as $M_{2} / \dot{M} \sim 3.48 \times 10^{7}$ yr (or $35 \mathrm{Myr}$ ) for equation (2) and $M_{2} / \dot{M} \sim 5.14 \times 10^{7}$ yr (or $51 \mathrm{Myr}$ ) for equation (3), while the timescale of the period decrease is $P / \dot{P} \sim 2.01 \times 10^{7}$ yr (or $20 \mathrm{Myr}$ ) without a sinusoidal term and $P / \dot{P} \sim 2.97 \times 10^{7}$ yr (or $30 \mathrm{Myr}$ ) with a sinusoidal term. The thermal timescale of the more massive component is $46.43 \times 10^{6} \mathrm{yr}$ (or $46 \mathrm{Myr}$ ). These timescales reveal that RW Dor is presently undergoing a slow mass transfer at the beginning stages of contact evolution with a high mass ratio, shallow contact configuration, and longterm decrease in the orbital period. In this way, the contact degree of the system will become higher and the system will evolve into a deeper contact binary. Another plausible 
Table 5. Times of light minima for RW Dor.

\begin{tabular}{|c|c|c|c|c|c|c|c|c|c|}
\hline $\begin{array}{c}\text { HJD } \\
(2400000+)\end{array}$ & $\begin{array}{l}\text { Error } \\
\text { (d) }\end{array}$ & Method & $\begin{array}{l}\text { Min. } \\
\text { (type) }\end{array}$ & Ref. & $\begin{array}{c}\text { HJD } \\
(2400000+)\end{array}$ & $\begin{array}{l}\text { Error } \\
\text { (d) }\end{array}$ & Method & $\begin{array}{l}\text { Min. } \\
\text { (type) }\end{array}$ & Ref.* \\
\hline 11298.835 & & pg & II & $(1)$ & 46681.7865 & 0.001 & pe & II & $(5)$ \\
\hline 14168.883 & & pg & II & (1) & 46690.7785 & 0.0001 & pe & I & (6) \\
\hline 15621.901 & & $\mathrm{pg}$ & II & (1) & 46695.7745 & 0.0001 & pe & II & (6) \\
\hline 16013.836 & & pg & II & (1) & 48500.0470 & & & I & (10) \\
\hline 16489.714 & & pg & II & (1) & 50559.9437 & 0.0004 & $\mathrm{ccd}$ & I & $(7)$ \\
\hline 17075.903 & & pg & I & (1) & 50560.0865 & 0.0003 & $\mathrm{ccd}$ & II & (7) \\
\hline 23784.600 & & pg & I & $(1)$ & 51158.5603 & 0.0002 & $\mathrm{ccd}$ & I & (7) \\
\hline 24172.537 & & pg & I & (1) & 51158.7027 & 0.0002 & $\mathrm{ccd}$ & II & (7) \\
\hline 30938.602 & & pg & I & (10) & 51505.6820 & 0.0002 & $\mathrm{ccd}$ & I & (7) \\
\hline 44313.581 & 0.001 & pe & II & $(2)$ & 51505.8252 & 0.0002 & $\mathrm{ccd}$ & II & (7) \\
\hline 44464.876 & 0.001 & pe & II & (2) & 51548.5020 & & $\mathrm{ccd}$ & I & (10) \\
\hline 44581.7728 & 0.0008 & pe & I & $(2)$ & 51869.0760 & & $\mathrm{ccd}$ & I & (10) \\
\hline 44608.6063 & 0.0005 & pe & I & (2) & 54036.5947 & & $\mathrm{ccd}$ & I & (10) \\
\hline 44608.7488 & 0.0006 & pe & II & (2) & 54036.7411 & & $\mathrm{ccd}$ & II & (10) \\
\hline 44609.6063 & 0.0008 & pe & II & (2) & 54037.5995 & & $\mathrm{ccd}$ & II & (10) \\
\hline 44609.7493 & 0.0005 & pe & I & (2) & 54037.7384 & & $\mathrm{ccd}$ & I & (10) \\
\hline 44610.7487 & 0.0009 & pe & II & (2) & 54041.7335 & & $\mathrm{ccd}$ & I & (10) \\
\hline 44825.8462 & 0.0008 & pe & I & (3) & 54049.5878 & & $\mathrm{ccd}$ & II & (10) \\
\hline 44826.8442 & 0.0004 & pe & II & (3) & 54059.7177 & & $\mathrm{ccd}$ & I & (10) \\
\hline 44873.8038 & 0.0006 & pe & I & (3) & 54087.9783 & 0.0001 & pe & I & $(8)$ \\
\hline 44874.6594 & 0.0003 & pe & I & (3) & 54091.1189 & 0.0002 & pe & I & (8) \\
\hline 44874.8010 & 0.0004 & pe & II & (3) & 54095.1150 & 0.0001 & pe & I & $(8)$ \\
\hline 44958.5851 & 0.0004 & pe & I & (3) & 54107.6761 & & ccd & I & (10) \\
\hline 44961.5843 & 0.0006 & pe & II & (3) & 55904.66718 & 0.00005 & $\mathrm{ccd}$ & I & (9) \\
\hline 44961.7239 & 0.0006 & pe & I & (3) & 55906.80840 & 0.00005 & $\mathrm{ccd}$ & II & (9) \\
\hline 44962.5815 & 0.0007 & pe & I & (3) & 56950.7446 & & $\mathrm{ccd}$ & II & (10) \\
\hline 44962.7267 & 0.0005 & pe & II & (3) & 57112.6009 & 0.0001 & $\mathrm{ccd}$ & II & (9) \\
\hline 45021.6738 & 0.0003 & pe & I & (3) & 57118.5968 & 0.0001 & $\mathrm{ccd}$ & II & (9) \\
\hline 45049.5058 & 0.0006 & pe & II & (3) & 57446.7363 & 0.0001 & $\mathrm{ccd}$ & I & (9) \\
\hline 45049.6486 & 0.0008 & pe & I & (3) & 57447.5930 & 0.0001 & $\mathrm{ccd}$ & I & (9) \\
\hline 45050.6484 & 0.0003 & pe & II & (3) & 57644.8485 & 0.0002 & $\mathrm{ccd}$ & I & (9) \\
\hline 45076.4815 & 0.0004 & pe & I & (3) & 57645.8477 & 0.0001 & $\mathrm{ccd}$ & II & (9) \\
\hline 45370.6556 & 0.0001 & pe & II & (4) & 57661.8334 & 0.0002 & $\mathrm{ccd}$ & II & (9) \\
\hline 45370.6558 & 0.0003 & pe & II & (4) & 57686.6689 & 0.0003 & $\mathrm{ccd}$ & II & (9) \\
\hline 45370.6564 & 0.0004 & pe & II & (4) & 57686.8119 & 0.0002 & $\mathrm{ccd}$ & I & (9) \\
\hline 45376.6502 & 0.0001 & pe & II & (4) & 57720.6390 & 0.0002 & $\mathrm{ccd}$ & II & (9) \\
\hline 45376.6507 & 0.0002 & pe & II & (4) & 57721.6383 & 0.0002 & $\mathrm{ccd}$ & I & (9) \\
\hline 45376.6517 & 0.0002 & pe & II & (4) & 57826.5461 & 0.0002 & $\mathrm{ccd}$ & II & (9) \\
\hline 46680.7878 & 0.001 & pe & I & (5) & 57827.5454 & 0.0002 & $\mathrm{ccd}$ & I & (9) \\
\hline
\end{tabular}

*References: (1) Hertzsprung (1928), (2) Marton and Grieco (1981), (3) Grieco and Marton (1983), (4) Russo, Vittone, and Milano (1984), (5) Marton, Grieco, and Sistero (1989), (6) Kaluzny and Caillault (1989), (7) Ogloza and Zakrzewski (2004), (8) Marino et al. (2007), (9) the present authors, (10) $\langle$ http://var.astro.cz/ocgate $\rangle$.

explanation for the long-term decrease in period is AML via magnetic stellar wind, which can be determined by the following equation given by Bradstreet and Guinan (1994):

$$
\begin{aligned}
\dot{P} \approx & -1.1 \times 10^{-8} q^{-1}(1+q)^{2}\left(M_{1}+M_{2}\right)^{-5 / 3} k^{2} \\
& \times\left(M_{1} R_{1}^{4}+M_{2} R_{2}^{4}\right) P^{-7 / 3},
\end{aligned}
$$

where $k^{2}$ is the gyration constant ranging from 0.07 to 0.15 for solar-type stars. By adopting a value of $k^{2}=0.1$ (Bradstreet \& Guinan 1994), the rate of orbital period decrease due to AML can be computed as $d P / d t$ $=-33.2 \times 10^{-9} \mathrm{dyr}^{-1}:$ in this case the timescale of period decrease is $P / \dot{P} \sim 8.598 \times 10^{6} \mathrm{yr}$ (or $8.6 \mathrm{Myr}$ ) which is about two times shorter than the timescale from equation (2) and three times shorter than that from equation (3). This means that the conservative mass transfer may not satisfactorily explain the secular decrease in period, or the mass transfer may be dynamical (Qian \& Zhu 2002). 

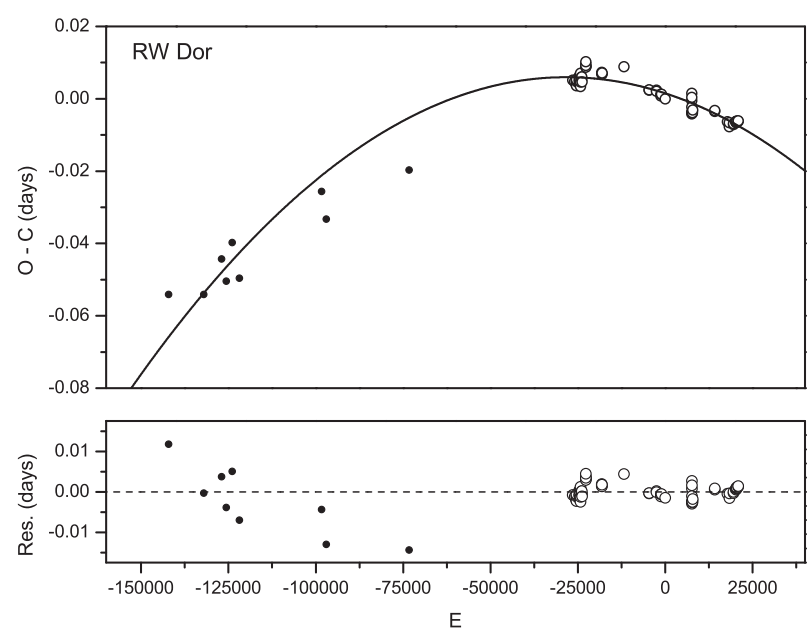

Fig. 2. $(O-C)$ curve. The dots before $E=-50000$ refer to photographic data (pg) and the open circles after $E=-50000$ refer to photoelectric (pe) and CCD data. The solid line in the upper panel was computed by using the quadratic term in equation (2), and this downward parabolic curve suggests a long-term decrease in period. The residuals from equation (2) are plotted in the lower panel.
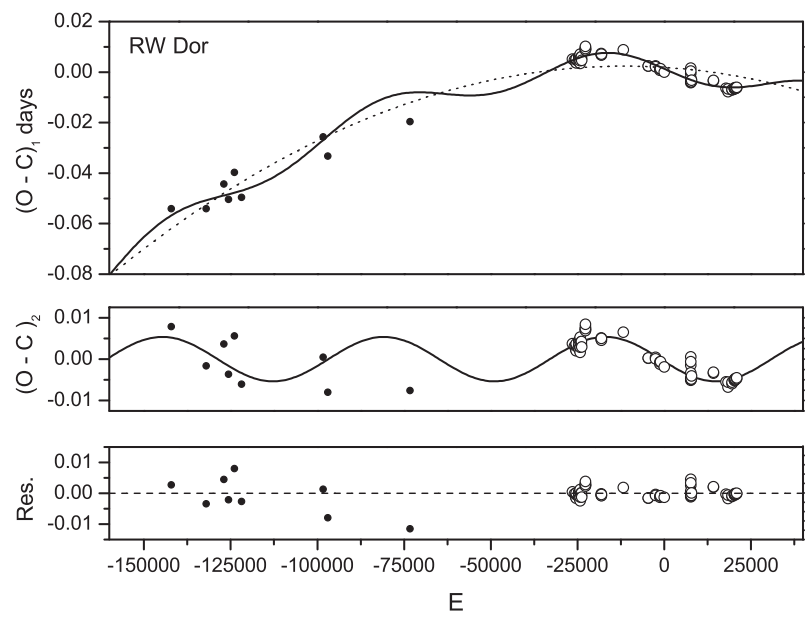

Fig. 3. $(O-C)_{1}$ diagram constructed by using the ephemeris in equation (1). The solid line in the upper panel refers to a combination of a long-term decrease in period and a small-amplitude cyclic variation, while the dashed line represents the long-term decrease of the orbital period. After subtracting the quadratic term, the result is displayed on the middle panel. The bottom panel shows the residuals for equation (3).

To explain this, Qian (2001a) proposed that the rate of AML is changed depending on the depth of overcontact. When the period decreases, the separation between the components becomes closer and the depth of contact increases, causing the common convective envelope (CCE) to become deeper and increase mixing in the CCE, which may result in AML at a lower rate (Vilhu 1981; Smith 1984). If the AML value is larger than the critical value of Rahunen (1981) and causes the orbital period to decrease, the evolution of RW Dor will be on the AML-controlled stage.
Table 6. Photometric solutions for the data in set- 1 and set-2 when $T_{1}=5560 \mathrm{~K}$.

\begin{tabular}{lcc}
\hline Parameters & set- 1 & set- 2 \\
\hline$T_{1}(\mathrm{~K})$ & 5560 (assumed) & fixed \\
$g_{1}=g_{2}$ & 0.32 (assumed) & fixed \\
$A_{1}=A_{2}$ & 0.50 (assumed) & fixed \\
$q\left(M_{2} / M_{1}\right)$ & 1.587 (assumed) & fixed \\
$T_{2}(\mathrm{~K})$ & $5287( \pm 10)$ & $5238( \pm 13)$ \\
$T_{1}-T_{2}(\mathrm{~K})$ & $273( \pm 5)$ & $322( \pm 6)$ \\
$T_{2} / T_{1}$ & $0.951( \pm 0.002)$ & $0.942( \pm 0.002)$ \\
$i\left({ }^{\circ}\right)$ & $77.2( \pm 0.1)$ & $76.9( \pm 0.2)$ \\
$\Omega_{\text {in }}$ & 3.091 & 3.091 \\
$\Omega_{\text {out }}$ & 2.732 & 2.732 \\
$\Omega_{1}=\Omega_{2}$ & $4.64( \pm 0.04)$ & $4.62( \pm 0.03)$ \\
$L_{1} /\left(L_{1}+L_{2}\right)(B)$ & $0.475( \pm 0.006)$ & $0.491( \pm 0.006)$ \\
$L_{1} /\left(L_{1}+L_{2}\right)(V)$ & $0.455( \pm 0.005)$ & $0.467( \pm 0.005)$ \\
$L_{1} /\left(L_{1}+L_{2}\right)\left(R_{\mathrm{C}}\right)$ & $0.444( \pm 0.005)$ & $0.455( \pm 0.005)$ \\
$L_{1} /\left(L_{1}+L_{2}\right)\left(I_{\mathrm{C}}\right)$ & $0.437( \pm 0.005)$ & $0.446( \pm 0.004)$ \\
$r_{1}($ pole $)$ & $0.3222( \pm 0.0013)$ & $0.3242( \pm 0.0014)$ \\
$r_{1}($ side $)$ & $0.3378( \pm 0.0014)$ & $0.3402( \pm 0.0015)$ \\
$r_{1}($ back $)$ & $0.3740( \pm 0.0015)$ & $0.3777( \pm 0.0018)$ \\
$r_{2}($ pole $)$ & $0.4057( \pm 0.0053)$ & $0.4069( \pm 0.0048)$ \\
$r_{2}($ side $)$ & $0.4309( \pm 0.0069)$ & $0.4326( \pm 0.0063)$ \\
$r_{2}($ back $)$ & $0.4635( \pm 0.0102)$ & $0.4658( \pm 0.0093)$ \\
$f$ & $11.5 \%( \pm 6.7 \%)$ & $15.0 \%( \pm 6.1 \%)$ \\
$\Sigma W_{i}(O-C)_{i}^{2}$ & 0.01641 & 0.01866 \\
\hline & &
\end{tabular}

Based on period studies by Qian (2001a), the evolution of RW Dor may be a combination of thermal relaxation oscillation (TRO) and AML changes through the variable depth of overcontact, e.g., V417 Aql (Qian 2003). In addition, the study by Marton, Grieco, and Sistero (1989) has shown that there is a hot spot on the cooler component located near the neck of the system, suggesting a secondary-toprimary mass transfer which is in good agreement with the long-term decrease in period. This indicates that RW Dor is in the transition phase to W UMa and at the beginning of the contact phase, being similar to VW Boot (Qian $\&$ Zhu 2002). If the orbital-period decrease is caused by losing angular momentum through magnetic braking, this is in agreement with the conclusion derived by Qian et al. (2017b, 2018) that some EW-type contact binaries were formed from short-period EA-type systems via Case A mass transfer (Vilhu 1982; Bradstreet \& Guinan 1994). Spectroscopic observations with LAMOST (Qian et al. 2017b, 2018) reveal that short-period $\mathrm{EW}$ binaries $(P<0.4 \mathrm{~d})$ have low metallicities, suggesting that EW-type binaries are old stellar populations and may be older than their long-period cousins. This means that they have a longer pre-contact phase (Qian et al. 2017b). Moreover, the evolution study of low-mass contact binaries by Stepien and Gazeas (2012) indicates that systems with a low total mass $\left(M<1.4 M_{\odot}\right)$ 

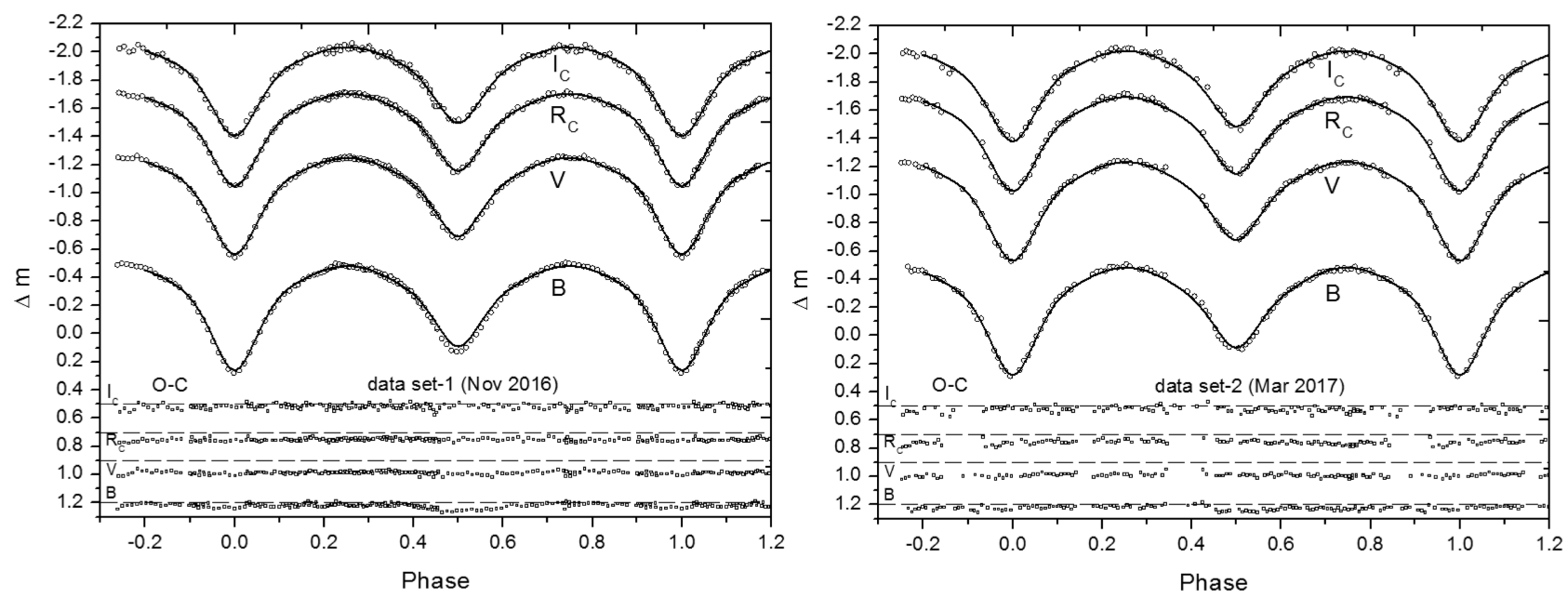

Fig. 4. Theoretical light curves (solid lines) computed by the W-D method compared with the observed light curves for the data in set-1 (left panel) and set-2 (right panel) without the third light and a spot. All theoretical light curves in $B V R_{C} I_{C}$ bands are fitted well to the observed light curves; this means that the physical parameters obtained from the light curve modeling are correct and reliable, except for the $B$-band light curves in set- 1 which do not fit well at the secondary minimum.
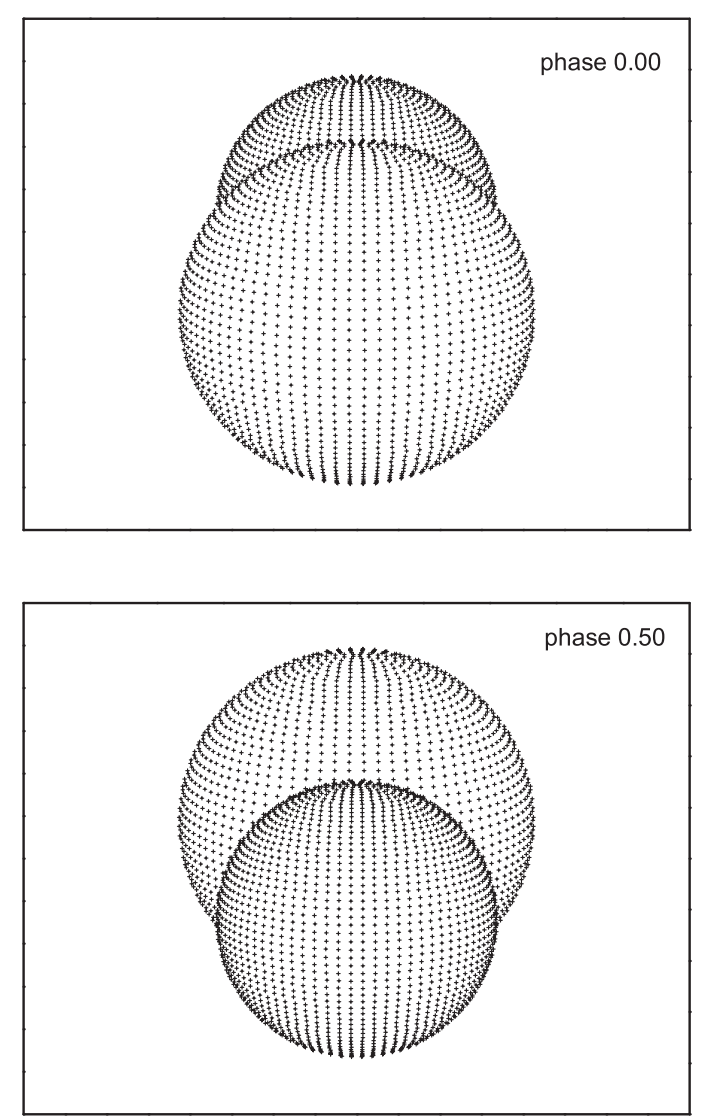

Fig. 5. Geometrical structures at phases of $0.00,0.25,0.50$, and 0.75 .

and a short orbital period $(P<0.3 \mathrm{~d})$ have a long precontact phase that lasts for $8-9 \mathrm{Gyr}$, while the contact phase takes only about 0.8 Gyr with a low mass transfer rate. The situation of RW Dor is in good agreement with the conclusions proposed by Stepien and Gazeas (2012) and Qian
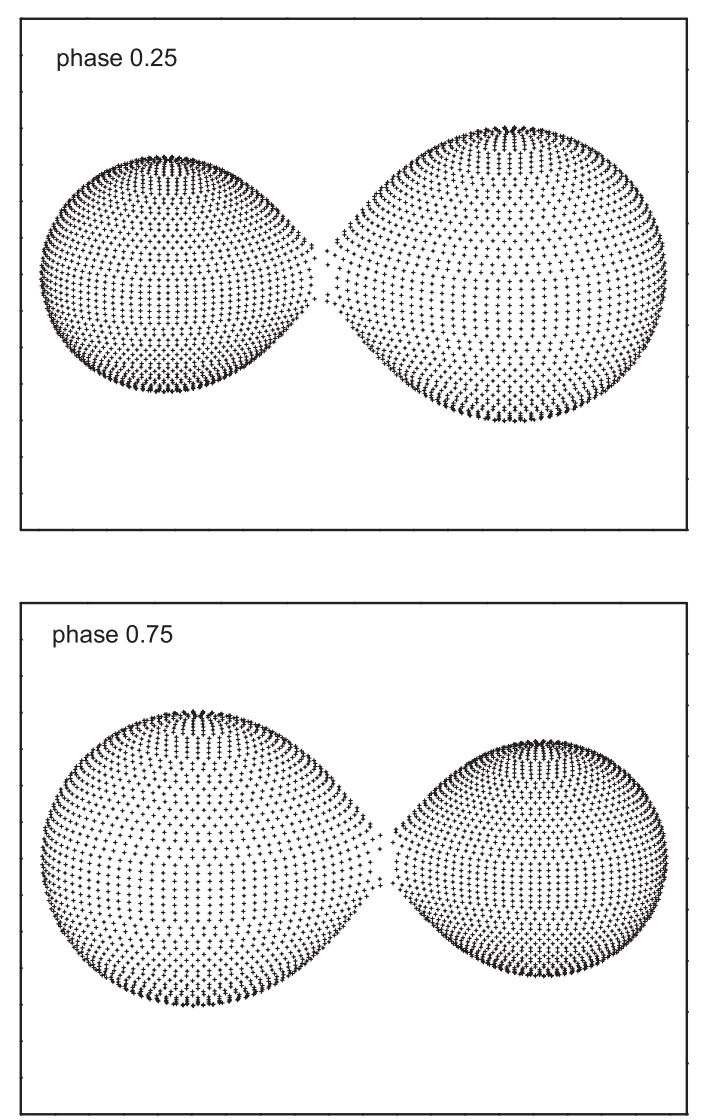

et al. (2017b, 2018) that a shallow-contact binary with a short period $(P \sim 0.285 \mathrm{~d})$, low total mass $\left(M \sim 1.34 M_{\odot}\right)$, and long-term decrease in period is a newly formed contact binary, which is similar to V524 Mon (He et al. 2012), MR Com (Qian et al. 2013b), BI Vul (Qian et al. 2013a), 
and CSTAR 038663 (Qian et al. 2014), at the beginning state of the contact phase or recently evolved into a contact configuration after it spent a long time in the pre-contact phase. In addition, the absolute parameters of RW Dor are quite close to V336 TrA (Kriwattanawong et al. 2018, their figure 4), suggesting that the more massive component (the secondary) of RW Dor will locate near the ZAMS (the zeroage main sequence), while the location of the less massive one should be close to the TAMS (the terminal-age main sequence). This means that the less massive one has evolved to reach the TAMS, whereas the more massive one has not evolved. This may be due to the mass transfer between the components.

In addition, a cyclic oscillation superimposed on a secular term is often found in W UMa-type binaries (see Qian 2001b, 2002). Furthermore, a study of the light travel-time effect in short-period eclipsing binaries by Li et al. (2018b) indicates that the frequency of third bodies found in contact binaries with a period shorter than $0.3 \mathrm{~d}$ reaches a value of $0.65(65 \%)$ in their samples of 542 eclipsing binaries. Therefore, it is possible that there may exist a periodic variation superimposed on a secular decrease in period in the $\mathrm{O}-\mathrm{C}$ curve of RW Dor, even weak evidence as explained in section 3. If we assume that the periodic change in the $O-C$ curve exists, by analysis of equation (3) the sinusoidal term reveals a cyclic change with a semi-amplitude of $0.0054 \mathrm{~d}$ and a period of $49.92 \mathrm{yr}$. The periodic variations in W UMa binary stars are usually explained by two ideas: one is the Applegate mechanism (Applegate 1992) via magnetic activity cycles at one component or both, and the second idea is the light travel-time effect (Liao \& Qian 2010; Han et al. 2016) via the presence of a third body.

The Applegate mechanism suggests that the cyclic change is caused by magnetic activity-driven variations in the quadrupole moment of solar-type components. Because RW Dor consists of G4/5 V spectral type stars, it should show strong magnetic activity. If this is the case, the quadrupole moment of the binary star can be determined from the equations given by Rovithis-Livaniou et al. (2000) and Lanza and Rodono (2002):

$$
\Delta P=\sqrt{\left[1-\cos \left(2 \pi P / P_{3}\right)\right]} \times A_{3},
$$

and

$\frac{\Delta P}{P}=-9 \frac{\Delta Q}{M a^{2}}$,

where $A_{3}$ is the amplitude of the sinusoidal term in equation (3), $P_{3}$ is the magnetic activity period, $R$ is the radius of the active star, and $a$ is the separation. The result is $\Delta P / P \sim 1.317 \times 10^{-6}$, and the quadruple moment for the primary star is $\Delta Q_{1}=3.07 \times 10^{48} \mathrm{~g} \mathrm{~cm}^{2}$ and that for the
Table 7. Parameters of the tertiary component for RW Dor.

\begin{tabular}{lclc}
\hline Parameter & Value & Error & Units \\
\hline$P_{3}$ & 49.9207 & 0.0003 & $\mathrm{yr}$ \\
$A_{3}$ & 0.0054 & 0.0001 & $\mathrm{~d}$ \\
$a_{12}^{\prime} \sin i^{\prime}$ & 0.929 & 0.026 & $\mathrm{au}$ \\
$f(m)$ & 0.00032 & 0.00003 & $M_{\odot}$ \\
$e_{3}$ & 0.0 & assumed & - \\
$M_{3}\left(i^{\prime}=90^{\circ}\right)$ & 0.087 & 0.002 & $M_{\odot}$ \\
$a_{3}\left(i^{\prime}=90^{\circ}\right)$ & 14.33 & 0.58 & $\mathrm{au}$ \\
\hline
\end{tabular}

secondary star is $\Delta Q_{2}=4.84 \times 10^{48} \mathrm{~g} \mathrm{~cm}^{2}$. These values for both components are lower than the typical values for an active contact binary, which range from $10^{51}$ to $10^{52} \mathrm{~g} \mathrm{~cm}^{2}$. Thus, the cyclic oscillation in figure 3 cannot be interpreted as the result of the Applegate mechanism. Furthermore, no spot activity cycles or light curve variations were found during the observations. The light curves may be stable for years (see figure 1) compared with the previous publications, which suggests that there is a very weak magnetic activity cycle at that time. Therefore, this period modulation may not be caused by a magnetic activity cycle that happens normally in single solar-type stars. There are many contact systems in which periodic variations cannot be explained by the Applegate mechanism, as discussed by Qian et al. (2013a), but the most probable reason causing the cyclic changes for those binaries is the light travel-time effect due to perturbations from a third body (Irwin 1952; Borkovits \& Hegedues 1996; Liao \& Qian 2010).

Therefore, a plausible idea for the cyclic period change is the light travel-time effect via the presence of a third body. By assuming that the tertiary component is moving in a circular orbit, the value of $a_{12}^{\prime} \sin i^{\prime}$ is computed as $0.929 \mathrm{au}$ by using the relation $a_{12}^{\prime} \sin i^{\prime}=A_{3} \times c$, where $A_{3}$ is the semi-amplitude of the $O-C$ oscillation, $c$ is the speed of light, and $i^{\prime}$ is the orbital inclination of the third component. Thus, the mass function, the masses, and the orbital radii of the third component in different inclination values can be determined by solving the following equation:

$f(m)=\frac{4 \pi^{2}}{G P_{3}^{2}} \times\left(a_{12}^{\prime} \sin i^{\prime}\right)^{3}=\frac{\left(M_{3} \sin i^{\prime}\right)^{3}}{\left(M_{1}+M_{2}+M_{3}\right)^{2}} ;$

the corresponding values are displayed in table 7. The mass function of the third body can be derived as $f(m)$ $=0.00032( \pm 0.00003) M_{\odot}$. The mass of the third body is $M_{3} \sin i^{\prime}=0.087( \pm 0.002) M_{\odot}$ and the orbital radius is $a_{3}=14.33$ au. If the minimum mass of the third body is $0.087 \mathrm{M}_{\odot}$, the third body will be a very low-mass star, red dwarf, or M-type star with extremely small luminosity and hence is difficult to detect. Since no third light was reported in the photometric studies (Marino et al. 2007; Deb \& Singh 2011) and no spectral lines of a third body were 
Table 8. Parameters of marginal contact binaries (W-type systems).

\begin{tabular}{|c|c|c|c|c|c|c|c|c|c|c|c|c|}
\hline Star & Sp. & $\begin{array}{l}\text { Period } \\
\text { (d) }\end{array}$ & $1 / q$ & $\begin{array}{c}f \\
(\%)\end{array}$ & $\begin{array}{c}d P / d t \\
\left(\times 10^{-} 8 \mathrm{dyr}^{-1}\right)\end{array}$ & Cyclic & $l_{3}$ & $\begin{array}{c}M_{1} \\
\left(M_{\odot}\right)\end{array}$ & $\begin{array}{c}M_{2} \\
\left(M_{\odot}\right)\end{array}$ & $\begin{array}{c}M_{3} \\
\left(M_{\odot}\right)\end{array}$ & Activities & Ref.* \\
\hline KIC 9532219 & G9 & 0.1981 & 0.833 & - & -52.7 & yes & $76 \%$ & - & - & 0.09 & spot & (1) \\
\hline CC Com & $\mathrm{K} 4 / 5$ & 0.2207 & 0.527 & 16.7 & -20.0 & yes & - & 0.37 & 0.71 & 0.066 & - & (2) \\
\hline BI Vul & K3 & 0.2518 & 0.964 & 8.7 & -9.5 & yes & no & 0.72 & 0.75 & 0.30 & spot & (3) \\
\hline V336 TrA & $\mathrm{K} 1$ & 0.2668 & 0.716 & 15.7 & - & - & - & 0.65 & 0.91 & - & spot & (4) \\
\hline CSTAR 038663 & $\mathrm{~K} 3$ & 0.2671 & 0.893 & 10.6 & - & yes & $<1 \%$ & 0.72 & 0.81 & $0.63 / 2.02$ & spot/flares & (5) \\
\hline BM UMa & $\mathrm{K}$ & 0.2712 & 0.540 & 17.0 & -7.49 & yes & - & 0.50 & 0.92 & 0.25 & - & (6) \\
\hline BX Peg & G5 & 0.2804 & 0.372 & 23.1 & -9.84 & yes & no & 0.38 & 1.02 & 0.22 & spot & (7) \\
\hline GSC $2765-0348$ & G4 & 0.2835 & 0.313 & 34.0 & - & - & - & - & - & - & 2 spots & (8) \\
\hline V524 Mon & G5 & 0.2836 & 0.476 & 7.7 & -.015 & yes & no & 0.50 & 1.10 & 0.26 & no & (9) \\
\hline LO Com & K0 & 0.2864 & 0.404 & 3.2 & -11.8 & yes & no & 0.32 & 0.79 & - & no & $(10)$ \\
\hline GSC 3526-01995 & $\mathrm{K} 2$ & 0.2922 & 0.351 & 18.2 & - & yes & no & 0.28 & 0.80 & 0.57 & spot & (11) \\
\hline IK Boo & G2 & 0.3031 & 0.873 & 2.2 & -21.7 & yes & - & 0.86 & 0.99 & 0.21 & spot & (12) \\
\hline V2284 Cyg & G7 & 0.3069 & 0.345 & 39.2 & -29.7 & yes & no & 0.30 & 0.86 & 0.036 & no & (13) \\
\hline TY Boo & G3 & 0.3171 & 0.466 & 12.0 & -2.99 & yes & - & 0.53 & 1.14 & 0.49 & - & (14) \\
\hline V1007 Cas & K0 & 0.3320 & 0.297 & 8.1 & -17.8 & - & $1.1 \%$ & 0.34 & 1.14 & - & spot & $(15)$ \\
\hline V781 Tau & G0 & 0.3449 & 0.453 & 21.6 & -6.01 & yes & no & 0.71 & 1.57 & 0.16 & spot & (16) \\
\hline V396 Mon & F8 & 0.3963 & 0.392 & 18.9 & -8.57 & yes & no & 0.36 & 0.92 & 0.31 & - & (17) \\
\hline PP Lac & G6 & 0.4012 & 0.435 & 23.9 & - & yes & no & 0.51 & 1.18 & 0.21 & no & (18) \\
\hline MR Com & F5 & 0.4127 & 0.256 & 10.0 & -53.0 & yes & $<1 \%$ & 0.36 & 1.40 & 0.18 & no & (19) \\
\hline RW Dor & G4/5 & 0.2854 & 0.630 & $>10$ & -1.42 & unclear & no & 0.52 & 0.82 & unclear & no & (20) \\
\hline
\end{tabular}

*References: (1) Lee et al. (2016), (2) Yang et al. (2009a), (3) Qian et al. (2013a), (4) Kriwattanawong et al. (2018), (5) Qian et al. (2014), (6) Yang, Wei, and Nakajima (2009b), (7) Lee et al. (2004, 2009), (8) Samec et al. (2012), (9) He et al. (2012), (10) Zhang, Han, and Liu (2016), (11) Liao, Qian, and Liu (2012), (12) Kriwattanawong, Sanguansak, and Maungkorn (2017), (13) Wang et al. (2017), (14) Yang et al. (2007), (15) Li et al. (2018a), (16) Li et al. (2016), (17) Liu et al. (2011), (18) Qian, Zhu, and Boonruksar (2005), (19) Qian et al. (2013b), (20) the present study.

found (Rucinski et al. 2007), to check the third body we also searched for the third light during the photometric solution, but the results showed negative values which mean that the contribution of the third light is very small compared with the total light of the system. Since its minimum mass $\left(i^{\prime} \sim 90^{\circ}\right)$ is small and it is located very far $(\sim 14.33 \mathrm{au})$ from the central binary system, it may not play an important role in the origin and evolution of the central binary by removing angular momentum from the inner system via the Kozai cycle (Kozai 1962). In this way, RW Dor will evolve normally into the contact phase without acceleration.

Based on section 3, there is very weak evidence for cyclic change in the $\mathrm{O}-\mathrm{C}$ analysis of figure 3 because of a few eclipse timings before 1948 with large scatter and a big gap of time between 1943 and 1980. However, almost contact binaries were found to be a triple or multiple system (Pribulla \& Rucinski 2006; D’Angelo et al. 2006; Rucinski et al. 2007), and one can see from table 6 that most of them have no third light in their light curves but show periodic variations in long-term period changes. Recently, a possible substellar object orbiting the solar-like contact binary V2284 Cyg was first reported by Wang et al. (2017), and also in the K-type shallow contact binary CC Com with very low mass $M_{3}=0.066 M_{\odot}$ by Yang et al. (2009a), and KIC 9532219 with $M_{3}=0.089 M_{\odot}$ by Lee et al. (2016) or
TX Cnc with $M_{3}=0.097 M_{\odot}$ by Liu et al. (2007). More recently, the eclipsing binary Kepler-503 has been found to be a brown dwarf or low-mass star with $M_{3}=0.075 M_{\odot}$ orbiting around a subgiant star (Cañas et al. 2018). Therefore, the existence of a third body in the system cannot be ruled out completely. To prove whether or not the invisible additional companion exists, long-term photometric monitoring with new eclipse timings and spectroscopic observations are required.

\section{Acknowledgments}

This work is supported by the National Natural Science Foundation of China (No. 11503077). We would like to thank Dr. Wiphu Rujopakarn and NARIT for time allocation to use PROMPT-8 for our observations. More CCD observations were obtained with the $2.15 \mathrm{~m}$ "Jorge Sahade" (JS) telescope at Complejo Astronomico El Leoncito (CASLEO), San Juan, Argentina. This research has made use of the SIMBAD online database, operated at CDS, Strasbourg, France, and NASA's Astrophysics Data System (ADS).

\section{References}

Applegate, J. H. 1992, ApJ, 385, 621

Borkovits, T., \& Hegedues, T. 1996, A\&AS, 120, 63

Bradstreet, D. H., \& Guinan, E. F. 1994, ASP Conf. Ser., 56, Interacting Binary Stars, ed. A. W. Shafter (San Francisco: ASP), 228 
Cañas, C. I., et al. 2018, ApJ, 861, L4

Cannon, A. J. 1921, Harvard Coll. Obs. Bull., 754

Cox, A. N. 2000, Allen's Astrophysical Quantities, 4th ed. (New York: Springer)

D’Angelo, C., van Kerkwijk, M. H., \& Rucinski, S. M. 2006, AJ, 132,650

Deb, S., \& Singh, H. P. 2011, MNRAS, 412, 1787

Duerbeck, H. W., \& Rucinski, S. M. 2007, AJ, 133, 169

Grieco, A., \& Marton, S. F. 1983, IBVS, 2436

Han, Z.-T., Qian, S.-B., Voloshina, I., Metlov, V. G., Zhu, L.-Y., \& Li, L.-J. 2016, Res. Astron. Astrophys., 16, 156

He, J., \& Qian, S. 2009, Ap\&SS, 321, 209

He, J.-J., Wang, J.-J., \& Qian, S.-B. 2012, PASJ, 64, 85

Hertzsprung, E. 1925, Bull. Astron. Inst. Netherlands, 2, 218

Hertzsprung, E. 1928, Bull. Astron. Inst. Netherlands, 4, 153

Hilditch, R. W., Hill, G., \& Bell, S. A. 1992, MNRAS, 255, 285

Høg, E., et al. 2000, A\&A, 355, L27

Irwin, J. B. 1952, ApJ, 116, 211

Kaluzny, J., \& Caillault, J.-P. 1989, Acta Astron., 39, 27

Kozai, Y. 1962, AJ, 67, 579

Kreiner, J. M. 2004, Acta Astron., 54, 207

Kriwattanawong, W., Sanguansak, N., \& Maungkorn, S. 2017, PASJ, 69, 62

Kriwattanawong, W., Sarotsakulchai, T., Maungkorn, S., Reichart, D. E., Haislip, J. B., Kouprianov, V. V., LaCluyze, A. P., \& Moore, J. P. 2018, New Astron., 61, 1

Kwee, K. K. 1958, Bull. Astron. Inst. Netherlands, 14, 131

Lanza, A. F., \& Rodono, M. 2002, AN, 323, 424

Layden, A. C., \& Broderick, A. J. 2010, PASP, 122, 1000

Leavitt, H. S. 1908, Annal. Harvard Coll. Obs., 60, 109

Lee, J. W., Hong, K., Koo, J.-R., \& Park, J.-H. 2016, ApJ, 820, 1

Lee, J. W., Kim, C.-H., Han, W.-Y., \& Kim, H.-I. 2004, MNRAS, 352,1041

Lee, J. W., Kim, S.-L., Lee, C.-U., \& Youn, J.-H. 2009, PASP, 121, 1366

Li, K., Gao, D.-Y., Hu, S.-M., Guo, D.-F., Jiang, Y.-G., \& Chen, X. 2016, Ap\&SS, 361, 63

Li, K., Hu, S., Guo, D., Jiang, Y., Gao, D., \& Chen, X. 2015a, New Astron., 41, 17

Li, K., Hu, S.-M., Guo, D.-F., Jiang, Y.-G., Gao, D.-Y., \& Chen, X. 2015b, New Astron., 34, 217

Li, K., Xia, Q.-Q., Hu, S.-M., Guo, D.-F., \& Chen, X. 2018a, PASP, 130, 074201

Li, M. C. A., et al. 2018b, MNRAS, 480, 4557

Liao, W.-P., \& Qian, S.-B. 2010, MNRAS, 405, 1930

Liao, W.-P., Qian, S.-B., \& Liu, N.-P. 2012, AJ, 144, 178

Liu, L., Qian, S.-B., Boonrucksar, S., Zhu, L.-Y., He, J.-J., \& Yuan, J.-Z. 2007, PASJ, 59, 607

Liu, L., Qian, S.-B., Liao, W.-P., He, J.-J., Zhu, L.-Y., Li, L.-J., \& Zhao, E.-G. 2011, AJ, 141, 44

Lucy, L. B., \& Wilson, R. E. 1979, ApJ, 231, 502

McLaughlin, D. B. 1927, AJ, 38, 45
Marino, B. F., Walker, W. S. G., Bembrick, C., \& Budding, E. 2007, PASA, 24, 199

Marton, F., \& Grieco, A. 1981, IBVS, 1960

Marton, S. F., Grieco, A., \& Sistero, R. F. 1989, MNRAS, 240, 931

O’Connell, D. J. K. 1951, Riverview Coll. Obs. Publ., 2, 85

Ogloza, W., \& Zakrzewski, B. 2004, IBVS, 5507

Pribulla, T., \& Rucinski, S. M. 2006, AJ, 131, 2986

Qian, S. 2001a, MNRAS, 328, 635

Qian, S. 2001b, MNRAS, 328, 914

Qian, S. 2002, A\&A, 384, 908

Qian, S. 2003, A\&A, 400, 649

Qian, S. B., \& Zhu, L. Y. 2002, ApJ, 568, 1004

Qian, S.-B., et al. 2013a, ApJS, 209, 13

Qian, S.-B., et al. 2014, ApJS, 212, 4

Qian, S.-B., et al. 2017a, ApJ, 848, 131

Qian, S.-B., He, J.-J., Zhang, J., Zhu, L.-Y., Shi, X.-D., Zhao, E.-G., \& Zhou, X. 2017b, Res. Astron. Astrophys., 17, 087

Qian, S.-B., Liu, N.-P., Liao, W.-P., He, J.-J., Liu, L., Zhu, L.-Y., Wang, J.-J., \& Zhao, E.-G. 2013b, AJ, 146, 38

Qian, S.-B., Zhang, J., He, J.-J., Zhu, L.-Y., Zhao, E.-G., Shi, X.-D., Zhou, X., \& Han, Z.-T. 2018, ApJS, 235, 5

Qian, S.-B., Zhu, L.-Y., \& Boonruksar, S. 2005, New Astron., 11, 52

Rahunen, T. 1981, A\&A, 102, 81

Rovithis-Livaniou, H., Kranidiotis, A. N., Rovithis, P., \& Athanassiades, G. 2000, A\&A, 354, 904

Rucinski, S. M., Pribulla, T., \& van Kerkwijk, M. H. 2007, 134, 2353

Russo, G., Vittone, A. A., \& Milano, L. 1984, A\&AS, 57, 69

Samec, R. G., Flaaten, D., Jaso, A., Oliver, B., \& Rehn, T. 2012, PASP, 124, 1025

Smith, R. C. 1984, QJRAS, 25, 405

Stepien, K., \& Gazeas, K. 2012, Acta Astron., 62, 153

Terrell, D., \& Wilson, R. E. 2005, Ap\&SS, 296, 221

Tian, X.-M., Zhu, L.-Y., Qian, S.-B., Li, L.-J., \& Jiang, L.-Q. 2018, Res. Astron. Astrophys., 18, 020

Van Hamme, W. 1993, AJ, 106, 2096

Van Hamme, W., \& Wilson, R. E. 2007, ApJ, 661, 1129

Vilhu, O. 1981, Ap\&SS, 78, 401

Vilhu, O. 1982, A\&A, 109, 17

Wang, J.-J., Jiang, L.-Q., Zhang, B., Zhao, S.-Q., \& Yu, J. 2017, PASP, 129, 124202

Wilson, R. E. 1990, ApJ, 356, 613

Wilson, R. E. 1994, PASP, 106, 921

Wilson, R. E. 2012, AJ, 144, 73

Wilson, R. E., \& Devinney, E. J. 1971, ApJ, 166, 605

Yang, Y.-G., Dai, J.-M., Yin, X.-G., \& Xiang, F.-Y. 2007, AJ, 134, 179

Yang, Y.-G., Lü, G.-L., Yin, X.-G., Zhu, C.-H., \& Nakajima, K. 2009a, AJ, 137, 236

Yang, Y.-G., Wei, J.-Y., \& Nakajima, K. 2009b, PASJ, 61, 13

Zhang, Y., Han, Q.-W., \& Liu, J.-Z. 2016, PASP, 128, 124201 